Case Report

\title{
Use of low-level laser therapy for oral lichen planus in children
}

\author{
Livia Assumpção Pedro ${ }^{a}$, Túlio Morandin Ferrisse ${ }^{\mathrm{a}}$, Darcy Fernandes ${ }^{\mathrm{a}}$, H.A. Silveira ${ }^{\mathrm{a}}$, \\ Rose Mara Ortega ${ }^{\mathrm{b}}$, Jorge Esquiche León ${ }^{\mathrm{c}}$, Andreia Bufalino ${ }^{\mathrm{a}, *}$ \\ a Oral Medicine, Department of Diagnosis and Surgery, São Paulo State University (Unesp), School of Dentistry, Araraquara, SP, Brazil \\ ${ }^{\mathrm{b}}$ Oral Medicine, Federal University of Juiz de Fora-UFJF/GV, Governador Valadares, Brazil \\ c Oral Pathology, Department of Stomatology, Public Oral Health, and Forensic Dentistry, School of Dentistry of Ribeirão Preto (FORP/USP), University of São Paulo, \\ Ribeirão Preto, SP, Brazil
}

\section{A R T I C L E I N F O}

\section{Keywords:}

Oral lichen planus

Pediatric patients

Low-level light therapy

Mucocutaneous immune-mediated disease

\begin{abstract}
A B S T R A C T
Oral lichen planus (OLP) is a chronic immune-mediated mucocutaneous disorder predominantly in white women after the fifth decade of life, rarely affecting children. Symptomatic OLP is usually treated with systemic and/or topical corticosteroids, but its prolonged use may cause several adverse effects. An eight-year-old girl presented bilateral white reticular plaques associated with atrophic areas involving the buccal and labial mucosa, and tongue dorsal surface with burning complaining. Medical history was non-contributory and an incisional biopsy was performed. Clinical and microscopic features were highly consistent with OLP diagnosis. Hence, 20 punctual low-level laser therapy (LLLT) sessions were performed, followed by significant clinical improvement and symptom discontinuation. We suggest that LLLT appears to be a successful treatment for childhood OLP, with good acceptance by pediatric patients.
\end{abstract}

\section{Introduction}

Lichen planus (LP) is a chronic immune-mediated mucocutaneous disease that may affect skin, scalp, nails and mucous membranes, especially the oral mucosa [1]. The disease is commonly found in middle aged and elderly patients, being more often in female patients and rarely found in children [2]. The etiology of LP is not well established, but studies demonstrate an abnormality of the $\mathrm{T}$ cell-mediated immune response, which can be caused by several factors like genetic polymorphism, unbalanced lifestyle and vitamin deficiencies. In some patients, it may be idiopathic [1,2]. Oral lichen planus (OLP) may present different clinical forms, but the most common are reticular, erythematous (erosive/ulcerative), and plaque type; whereas atrophic and bullous forms are less common [1,2]. Interestingly, two or more clinical forms may coexist in the same patient or it may change during the course of the disease. Corticotherapy is required for symptomatic patients but it may present important adverse effects [1,3,4]. The purpose of this study was to introduce a new approach to OLP treatment successfully with low-level laser therapy (LLLT) in a rare case of a pediatric patient.

\section{Case report}

An 8-year-old caucasian girl was referred for evaluation of multiple oral lesions complaining of an intense burning sensation on the tongue with 6 months of duration. The medical and family histories were noncontributory. Intraoral examination revealed multiple white reticular plaques with erythematous areas involving bilateral buccal mucosa, lower and upper labial mucosa, and dorsal surface of the tongue. The patient did not present extraoral involvement. There was no history of previous infections or another associated factor as amalgam restorations, flavorings, vaccinations or any medication. Complete blood count and serology for HBV e HCV were negative. An incisional biopsy on the dorsal surface of the tongue was performed. Microscopic analysis showed focal areas of hyperkeratinized epithelium and occasional areas of atrophic epithelium, serrated ridges, hydropic degeneration and a dense band-like lymphocytic infiltrate at the interface between the epithelium and the connective tissue. Based on the clinical and histopathological findings, the case was diagnosed as oral lichen planus OLP (Fig. 1). Considering the potential side effects of the conventional corticosteroid treatment, we used LLLT as an alternative treatment. The Photon Lase III equipment (PL7336, DMC, São Carlos - São Paulo, Brazil) used in the treatment was adjusted to red laser $(660 \mathrm{~nm})$, $100 \mathrm{~mW}$ potency, with energy density of $35 \mathrm{~J} / \mathrm{cm}^{2}, 7 \mathrm{~s}$ per site, and spot of $600 \mu \mathrm{m}$. The method of application was punctual, being $1 \mathrm{~cm}$ of distance between the points of application: 6 points over the lesion located on the tongue lesion and 3 points on each side of the buccal mucosa, and 1 point on each labial mucosa lesion. Each session was

\footnotetext{
* Corresponding author at: Department of Diagnosis and Surgery, Univ Estadual Paulista (UNESP), CEP 14801-903, Araraquara, São Paulo, Brazil.

E-mail address: andreiabufalino@foar.unesp.br (A. Bufalino).
} 


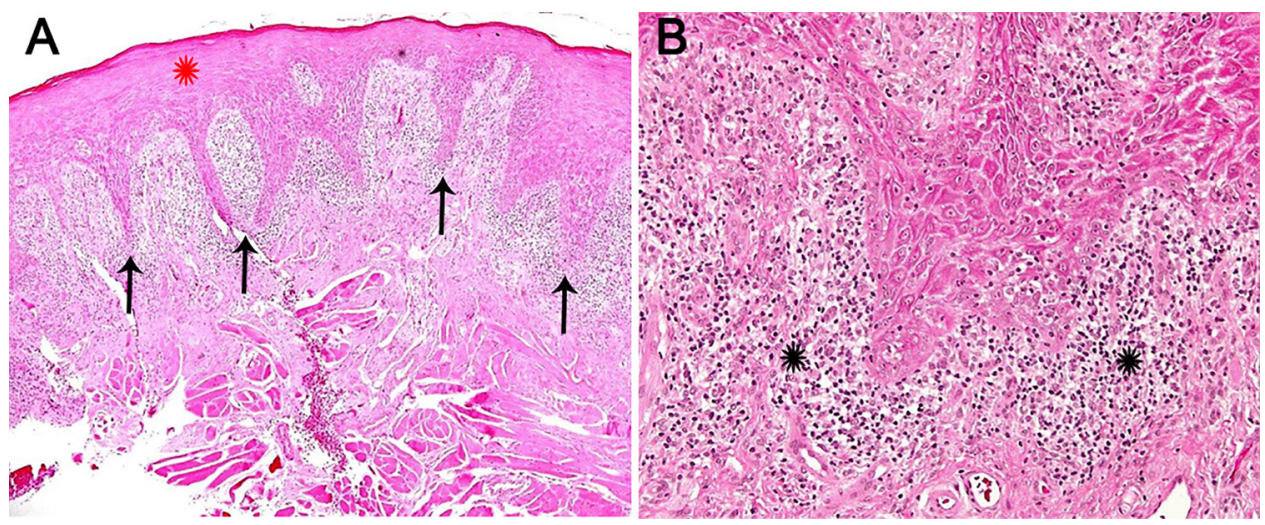

Fig. 1. Histopathological analysis revealed hyperkeratosis, serrated ridges (black arrows) and acanthosis (red star), as well as exocytosis, hydropic degeneration and subepithelial lymphocytic inflammatory infiltrate (black star) (Hematoxylin and eosin stain; original magnification, A: $\times 40$, B: $\times 100$ ). (For interpretation of the references to color in this figure legend, the reader is referred to the web version of this article).
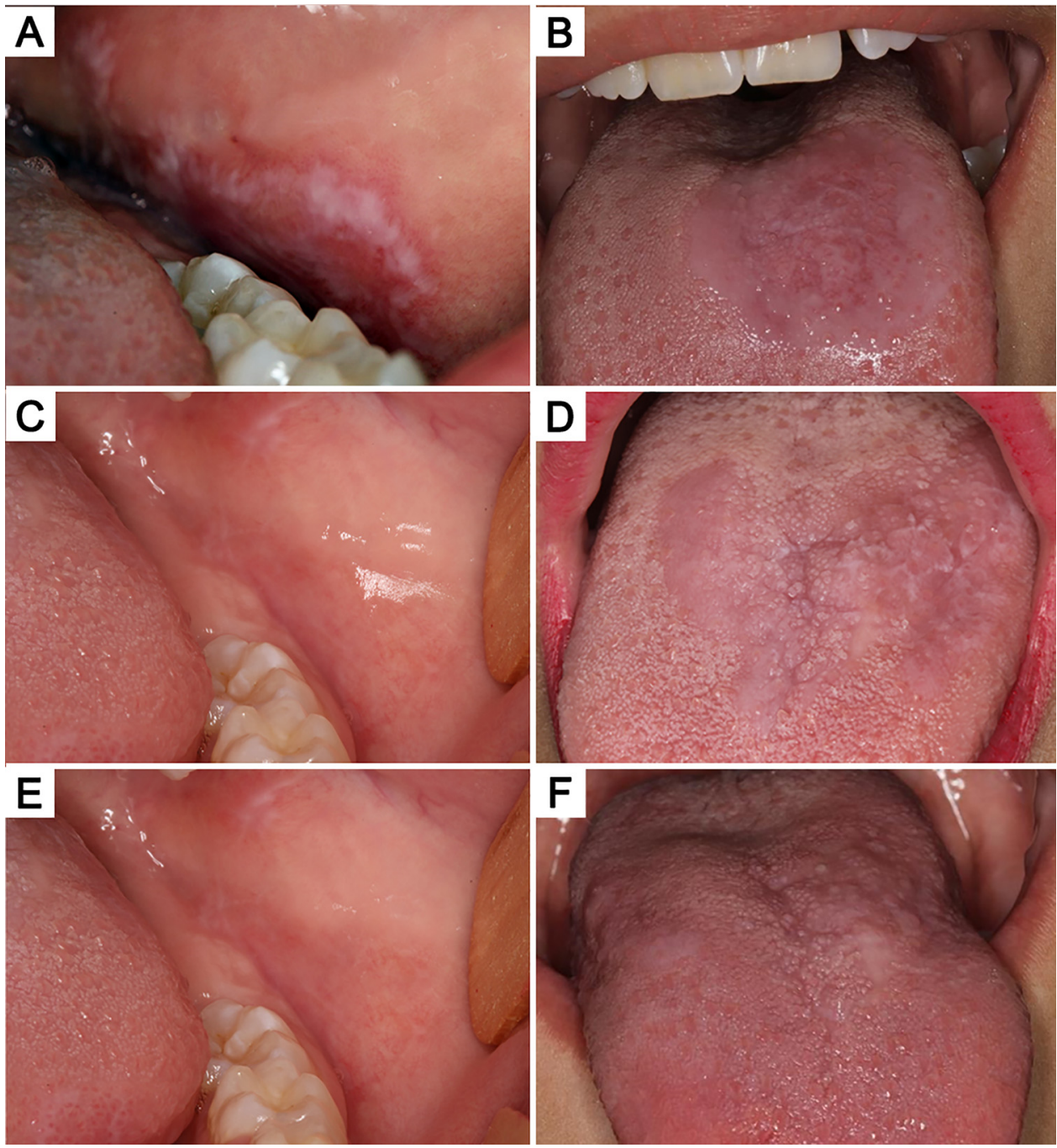

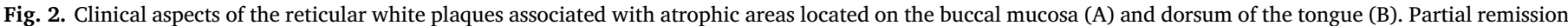
of the lesions and symptomatology after 8 sessions of LLLT (C and D). Complete remission of lesions and symptoms after 20 sessions of LLLT (E and F).

performed at $72 \mathrm{~h}$ intervals. After 9 laser therapy sessions, the patient reported significant improvement in burning sensation and after 20 sessions we could observe a sweetening of the white plaques and erosive areas, as well as improvement of symptoms (Fig. 2). The patient did not present any recurrence within 2 years of follow up (Fig. 3). No other long-term effects were noticed within this period.

\section{Discussion}

Reticular type of OLP with burning symptomatology involving buccal mucosa is the most frequent presentation in childhood OLP. The age of presentation occurs over 6 years, with a slight predilection for female. Only a few cases presented simultaneously cutaneous lesions and we have observed that most of cases used topical corticotherapy as main treatment for symptomatic patients. To the best of our knowledge, we report the first case of OLP in a pediatric patient treated successfully 

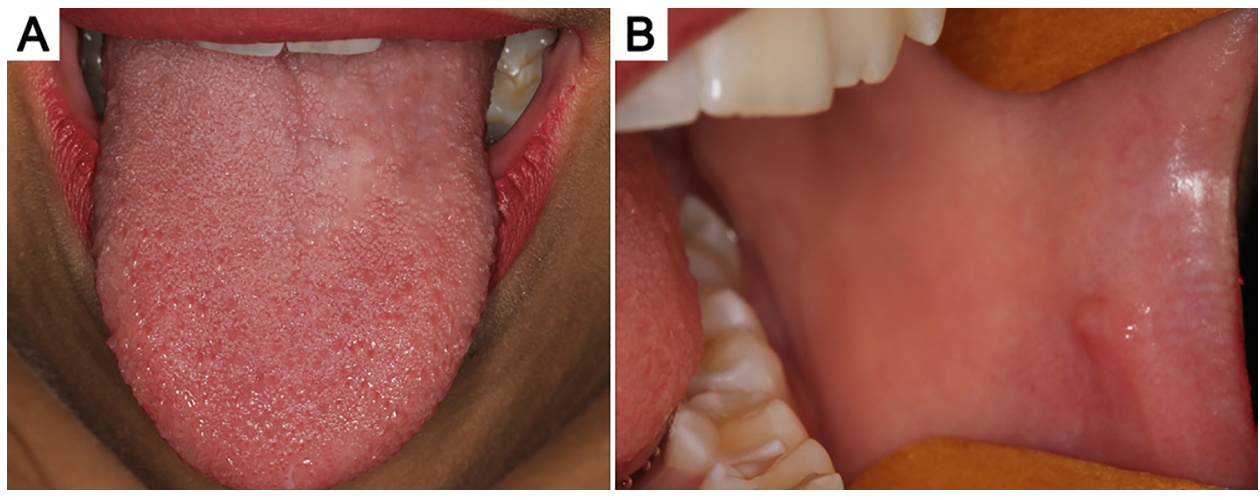

Fig. 3. Clinical presentation after 2 years of follow up. No recurrence was observed.

with LLLT.

The exact etiology of OLP is unknow. Some cases of childhood OLP are associated with the hepatitis B or C virus infection and the HBV vaccination. It appears to cause an improper cell-mediated autoimmune response [5,6]. Apart from conventional LP, there is a rare familial form more prevalent and more severe in the pediatric patients. It is an autosomal dominant disease and some specific types of HLA was already associated with this form of lichen planus [7].

OLP patients with symptomatic lesions usually need drug treatment with topical or systemic agents. Oftentimes, topical corticosteroids are effective in cases of symptomatic OLP, however, adverse effects may be observed. Topical corticosteroids may cause dermatologic and mucous issues such as atrophy, steroid acne, rosacea, perioral dermatitis, telangiectasia, masking of opportunistic infections, hypertrichosis and hypopigmentation [3]. They also can result in systemic adverse effects, involving the suppression of the hypothalamic-pituitary-adrenal axis, causing Cushing's Syndrome or growth delay in children [3]. Systemic corticosteroids as well may cause injuries like morphologic changes, hyperglycaemia, infections, delayed wound healing, increased blood pressure, among others [4].

Since no adverse effects of LLLT have been reported when correctly applied, the LLLT has been used for OLP treatment in adult patients [8]. It promotes primary and secondary physiologic effects on LLLT-treated tissues. In the primary effect, there are vasodilatation, improvement of blood flow, lymph drainage, cellular metabolism, neutrophil and fibroblast activation, and pain stimulation threshold. The secondary effect consists in aggregation of prostaglandins, immunoglobulins, cytokines, beta-endorphin and encephalin in the tissue, resulting in reduction of inflammation and pain [9]. All these potential biostimulating effects give us a reducing of the pharmacological support and its possible negative repercussion in our patients [9]. Moreover, it is showed in a recent systematic review that LLLT is effective in management of symptomatic OLP and may be used as an alternative to corticosteroids in adult patients [10].

In conclusion, we propose the use of LLLT as the treatment of choice to OLP in pediatric patients considering its notable success in the symptoms control, high acceptance by these patients and absence of side effects compared with conventional corticotherapy. However, further studies, as controlled randomized clinical trials with large sample sizes, are required to confirm the findings of this case report.

\section{Ethical approval}

The ethics committee approval was not necessary for this type of work.

\section{Conflicts of interest}

The authors declare no conflicts of interest.

\section{Funding}

No specific grant was received from funding agencies in the public, commercial, or not-for-profit sectors.

\section{References}

[1] Scully C, Carrozzo M. Oral mucosal disease: lichen planus. Br J Oral Maxillofac Surg 2008;46:15-21.

[2] Mohan Das U, Jp B. Oral lichen planus in children. Int J Clin Pediatr Dent 2009;2:49-51.

[3] Schackert C, Korting HC, Schäfer-Korting M. Qualitative and quantitative assessment of the benefit-risk ratio of medium potency topical corticosteroids in vitro and in vivo: characterisation of drugs with an increased benefit-risk ratio. BioDrugs 2000; $13: 267-77$.

[4] Poetker DM, Reh DD. A comprehensive review of the adverse effects of systemic corticosteroids. Otolaryngol Clin N Am 2010;43:753-68.

[5] Chatterjee K, Bhattacharya S, Mukherjee CG, Mazumdar A. A retrospective study of oral lichen planus in paediatric population. J Oral Maxillofac Pathol 2012;16:363-7. http://dx.doi.org/10.4103/0973-029X.102486.

[6] Cascone M, Celentano A, Adamo D, Leuci S, Ruoppo E, Mignogna MD. Oral lichen planus in childhood: a case series. Int J Dermatol 2017;56:641-52. http://dx.doi. org/10.1111/ijd.13571.

[7] Pandhi D, Singal A, Bhattacharya SN. Lichen planus in childhood: a series of 316 patients. Pediatr Dermatol 2014;31:59-67. http://dx.doi.org/10.1111/pde.12155.

[8] Cotler HB, Chow RT, Hamblin MR, Carroll J. The use of low level laser therapy (LLLT) for musculoskeletal pain. MOJ Orthop Rheumatol 2015;2. http://dx.doi.org/ 10.15406/mojor.2015.02.00068.

[9] Cafaro A, Arduino PG, Massolini G, Romagnoli E, Broccoletti R. Clinical evaluation of the efficiency of low-level laser therapy for oral lichen planus: a prospective case series. Lasers Med Sci 2014;29:185-90.

[10] Al-Maweri SA, Kalakonda B, Al-Soneidar WA, Al-Shamiri HM, Alakhali MS, Alaizari $\mathrm{N}$. Efficacy of low-level laser therapy in management of symptomatic oral lichen planus: a systematic review. Lasers Med Sci 2017;32:1429-37. http://dx.doi.org/ 10.1007/s10103-017-2233-2237. 